\title{
Counting of oscillatory modes of valence quarks forming qqq baryons for 3 quark flavors $\mathrm{u}, \mathrm{d}, \mathrm{s}$
}

\author{
Sonia Kabana ${ }^{1,2, a}$ and Peter Minkowski ${ }^{2, b}$ \\ ${ }^{1}$ University of Nantes, Nantes, France \\ ${ }^{2}$ Albert Einstein Center for Fundamental Physics - ITP, University of Bern, Bern, Switzerland
}

\begin{abstract}
We present a selection the unique properties of oscillatory modes of $N_{f l}=3$ light quarks $-u, d, s^{-}$, using the $\mathrm{SU}\left(2 N_{f l}=6\right) \times \mathrm{SO} 3(\vec{L})$ broken symmetry classification. $\vec{L}=\sum_{n=1}^{N} \vec{L}_{n}$ stands for the space rotation group generated by the sum of the three individual angular momenta of quarks in their c.m. system. The baryonic multiplets are shown to emerge from the picture of oscillating quarks in 3 space dimensions in the center of mass system of the baryons. All oscillatory modes are fully relativistic with a finite number of oscillators and ths is forming the unique harmonic oscillators with these properties. The density of states as a function of mass-square is calculated. This estimate is of relevance for the accounting of the missing states of unobserved hadrons, as the here estimated baryonic multiplets include both the observed and the unobserved (or "missing") hadrons. The estimate is conceptually different from Hagedorn's model and is based on field theory of QCD.
\end{abstract}

\section{Introduction}

The underlying field theory of strong interaction, QCD, assigns a local spin $1 / 2$ field to every valence quark and antiquark with the quantum numbers color and flavor, interacting locally with spin 1 color octet gauge fields, called gluons. The gauge interactions of the gluons are exactly conserved gauge transformations based on the local gauge group SU(3) of color. The field theoretic concepts were developed as core topics of an in depth investigation together with the oscillatory modes of quarks suggested by linear Regge trajectories [1].

The linear Regge trajectories (e.g. ref. [1] and [2]) with exception of the pomeron, suggest oscillatory behaviour, whereas the difficulty to describe them is due to the fact that the field theory variables fall into the perturbatively non accessible domain. Lattice QCD in principle is set to solve the nonperturbative problems ref. [3] .

Within the theory of Quantum Chromo Dynamics the mesons and baryons are arranged in multiplets while it was already found since the mid-1960'ies that many states are missing refs. [4], [5], [6] in the sense that they have not been detected by experiments even though they are predicted by the quark model. The non observation of some states can be due for example to resonance states overlapping or being very wide. The missing states remain missing up to the present.

\footnotetext{
a e-mail: kabana@mail.cern.ch

be-mail: mink@itp.unibe.ch
} 
In this paper we are constructing the full theoretically expected multiplets of baryons including those measured and those that are missing, and count their states, based on the oscillatory modes for quarks and antiquarks.

The oscillatory modes of valence quarks and antiquarks in mesons and baryons were developed by one of us (P.M.) in CALTECH from 1975 onwards and published in ref. [1], ref. [7], ref. [8] . In reference [8] only non strange hadrons have been considered, which have been compared to a good compilation of missing states of non strange baryons, as can be found in PDG 1980. However there are missing states also among the strange hadrons ref. [9] or ref. [10] .

The main idea of this approach is to use relative coordinates and associated canonically conjugate momenta in the center of mass of $q \bar{q}^{\prime}$ and $q q^{\prime} q^{\prime \prime}$ systems to describe harmonic oscillations of the valence quarks and antiquarks, with full special relativistic invariance using the zero width approximation for resonances.

We are separating quark antiquark spins combining them to baryonic sub-multiplets.

Oscillator modes mean by definition gaussian wavefunction in the ground state, either in configuration space or in momentum space.

With respect to light quark masses u,d,s we take the chiral limit. Further the limit considered is the long range approximation between quarks and antiquarks which involves large main oscillatory quantum number $\mathrm{N}$.

The model is valid strictly for large $\mathrm{N}$. For small and finite $\mathrm{N}$ the multiplets are not degenerate for different I, J, P, C quantum numbers of particles, deviating from the large $\mathrm{N}$ asymptoytic behaviour.

Many modes are unstable against strong decays and are approximated in the zero width limit.

A perturbatively renormalizable field theory does not allow an exponential growth of mass- and/or masssquare-density of such states because it would lead to an infinite number of free constants, which render the theory non-renormalizable. In string theory there is an infinite number of independent oscillators (like many springs) one each for the ground frequency $\omega, 2 \omega, 3 \omega$ etc. This cannot be described by a field theory which contains only a finite number of degrees of freedom. The string theory allows a spectrum of density growing exponentially with mass(square).

The prediction of Hagedorn's bootstrap model [11], was an exponential spectrum of hadron resonances, which later was shown to be realized in string theory, refs. [12], [13], [14], [15]. In addition the limiting temperature resulting from the exponential hadron resonance spectrum in Hagedorn's model was associated with the critical temperature of the QCD phase transition between hadrons and partons [16] . However if a limiting temperature exists from the hadron spectrum this is not nessecarily associated with a phase transition.

In string theory there is a limiting temperature à la Hagedorn, which is determined from the string tension i.e. of the order of the Planck mass and it is not nessecarily associated with a phase transition. An exponentially growing spectrum of hadron resonances would not agree with the expectation for an underlying field theory of strong interactions.

The approximate use of a broken extended symmetry combining $S U 6=S U\left(2\left(\sum_{q} \vec{S}_{q}\right) \times 3{ }_{f l}\right)$ with $S O 3\left(\sum_{q} \vec{L}_{q}\right)$ was vigorously pursued up to the end of the 60 -ies, whence the three valence quarks wave function is conceived in their c.m. frame. Other orbital $q \bar{q}^{\prime}$ and 3-quark wave functions were also considered, as e.g. the $p_{z} \rightarrow \infty$ states, where a boosted $S U 6_{w}$ symmetry is introduced instead of $S U 6$. In this respect the main quantum number $\mathrm{N}$ was not related to genuine oscillator degrees of freedom but instead inferred from recurrences along Regge trajectories from the relation

$$
\alpha^{\prime} M^{2}(J)=J+J_{0} \text { with } \alpha^{\prime} \Delta M^{2}=\Delta J=\Delta N
$$

In eq. $1 \vec{J}=\vec{L}+\vec{S}$ denotes total angular momentum. 
There is some literature quoted in ref. [8], which is however very limited, and among the many papers on that matter we mention here few representative ones by J. Schwinger [4], R. Dashen and M. GellMann [5] and by G. Zweig [6], illustrating the search for local field variables underlying the strong interactions at that time.

Historically, one result from the investigations and efforts towards identifying the known baryon resonances, was the finding that several states were missing. The counting of oscillatory modes of baryons with light flavored $\mathrm{u}, \mathrm{d}, \mathrm{s}$ quarks, that we report here, is a new investigation.

The estimate and counting of oscillatory modes of mesons with light flavored $\mathrm{u}, \mathrm{d}$, s quarks and corresponding antiquarks has been published previously in ref. [17]. In the following we will elaborate on the oscillating modes of baryons and compare with the measured ones.

Oscillatory modes of quarks in baryons reveal many properties going beyond the quark model, e.g. the numbers of modes with fixed main quantum number $\mathrm{N}$.

We do not discuss here the techniques derived from Young tableaux, applied to

$\mathrm{SU}\left(2 N_{f l}=6\right) \times \mathrm{SO} 3(\vec{L})$. Instead let us quote the book by Hermann Weyl [18] .

\section{Oscillatory modes for $\mathrm{u}, \mathrm{d}, \mathrm{s}$ baryons}

\section{Dynamics - Poincaré-invariant harmonic oscillator modes for baryons in the space of barycentric coordinates for 3 valence quarks $u, d, s$}

Seen from the time line of the first years of the 70-th it took about 9 years until an explicit construction of oscillator modes of baryons exhibiting a density function

$$
\varrho_{n}\left(m^{2}\right)=\frac{\partial N\left(m^{2}\right)}{\partial m^{2}} ; N=m^{2} \cdot \alpha^{\prime}
$$

where $\alpha^{\prime}$ is the slope of Regge trajectories, and

$$
\begin{aligned}
& \varrho_{n}\left(m^{2}\right) \sim\left(\frac{m^{2}}{m_{0}^{2}}\right)^{u} \text { for } m \rightarrow \infty \\
& m_{0}, u: \text { characteristic parameters and } u=\text { positive integer }
\end{aligned}
$$

for valence quark (antiquark) configurations in mesons and baryons - ref. [8] - compatible with a finite number of degrees of freedom .

Figure 1 shows the logarithm of the density of states of baryons defined in formula 3 , together with the density of states of mesons from ref. [17], [19] as a function of the main quantum number $\mathrm{N}$ plus one. This calculation (shown here for $\mathrm{N}+1$ until 10) includes only oscillatory modes for $\mathrm{u}, \mathrm{d}, \mathrm{s}$ quarks and antiquarks for $q q q$ baryons and $q \bar{q}$ for mesons, i.e. not including exotics. The shown density of states is valid in the limit of large N. Furthermore the here derived formulae do not include the intercept and assume a universal slope of Regge trajectories. This does not affect the counting of the number of these states. We observe that the baryon density of states grows faster than the meson density and is always larger than the latter. The data used in Fig. 1 come from Table 1 below. 


\begin{tabular}{|c|c|c|}
\hline $\mathrm{N}$ & $\mathrm{z}$ (Meson) & $\mathrm{z}$ (Baryon) \\
\hline 0 & 36 & 56 \\
\hline 1 & 108 & 210 \\
\hline 2 & 216 & 1044 \\
\hline 3 & 360 & 2780 \\
\hline 4 & 540 & 5766 \\
\hline 5 & 756 & 12510 \\
\hline 6 & 1008 & 22366 \\
\hline 7 & 1296 & 38052 \\
\hline 8 & 1620 & 62826 \\
\hline 9 & 1980 & 97540 \\
\hline
\end{tabular}

Table 1: Density of states for each main quantum number $\mathrm{N}$ until $\mathrm{N}=9$, for mesons and baryons with $\mathrm{u}, \mathrm{d}, \mathrm{s}$ quarks and antiquarks.

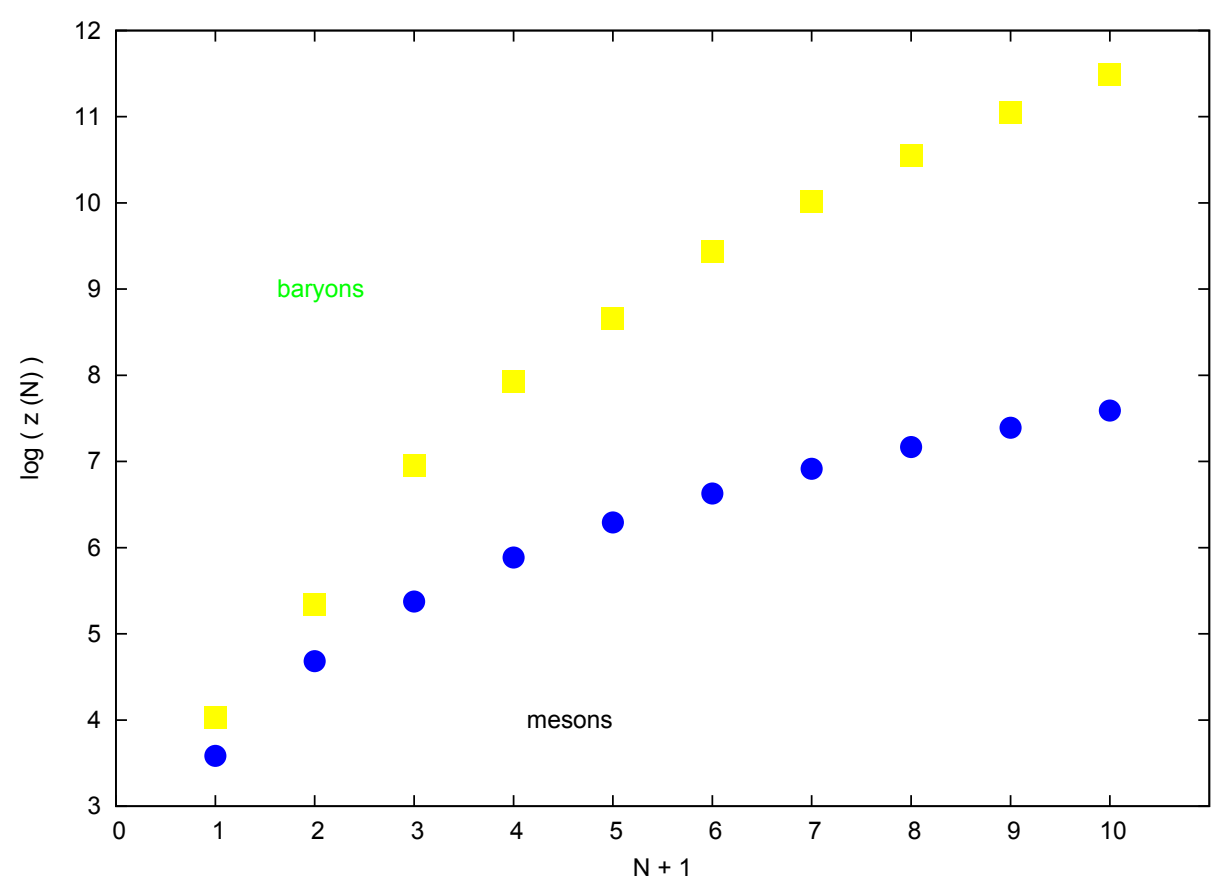

Figure 1. Fig. 1: Logarithm of the density of states of baryons defined in eq. 3 together with the density of states of mesons from [17] as a function of the main quantum number $\mathrm{N}$ plus one. 
We envisage baryons within QCD, allowing temporarily the local color gauge group to be generalized from 3 colors to $1<N_{c} \rightarrow N$ to simplify notation, whenever no confusion arises. Baryons are : fermions for $N$ odd, bosons for $N$ even . A concise sketch of barycentric canonically conjugate momentum and spatial coordinates - pairs of three vectors

$$
\left(\vec{\pi}_{v}, \vec{z}_{v}\right) ; v=1,2, \cdots, N
$$

follows .

\section{Barycentric coordinates in the overall c.m. system}

$$
\begin{aligned}
& \vec{\pi}_{1}=\frac{1}{\sqrt{2}}\left(\vec{p}_{1}-\vec{p}_{2}\right) \quad, \quad \vec{z}_{1}=\frac{1}{\sqrt{2}}\left(\vec{x}_{1}-\vec{x}_{2}\right) \\
& \vec{\pi}_{2}=\frac{1}{\sqrt{6}}\left(\vec{p}_{1}+\vec{p}_{2}-2 \vec{p}_{3}\right), \vec{z}_{2}=\frac{1}{\sqrt{6}}\left(\vec{x}_{1}+\vec{x}_{2}-2 \vec{x}_{3}\right) \\
& \vec{\pi}_{\nu}=(v(v+1))^{-1 / 2}\left(\begin{array}{cc}
\sum_{\alpha=1}^{v} & \vec{p}_{\alpha} \\
-v & \vec{p}_{v+1}
\end{array}\right), \vec{z}_{v}=(v(v+1))^{-1 / 2}\left(\begin{array}{cc}
\sum_{\alpha=1}^{v} & \vec{x}_{\alpha} \\
-v & \vec{x}_{v+1}
\end{array}\right) \\
& \vec{\pi}_{N-1}=\cdots \quad, \quad \vec{z}_{N-1}=\cdots \\
& \vec{\pi}_{N}=N^{-1 / 2} \sum_{\alpha=1}^{N} \vec{p}_{\alpha} \rightarrow 0, \vec{z}_{N}=N^{-1 / 2} \sum_{\alpha=1}^{N} \vec{x}_{\alpha} \rightarrow 0
\end{aligned}
$$

The last line in eq. 5 refers to c.m. momenta and positions, both fixed to vanish, they do not represent genuine degrees of freedom .

\subsection{Completing canonically conjugate variables in the pairs in $\mathcal{L}_{N}, t$}

In the local Lagrangean density setting of QCD the external quark masses, denoted $M_{q}$, appropriate multipliers of the scalar densities $\bar{q} q$ composing the mass term, enter in the form, also discussed recently in refs. $20-[20]-$ and $21-[21]-$

$$
-\mathcal{L}_{q-\text { mass }}=\sum_{\text {flavors }} \frac{z_{q}}{z_{M}} M_{q} \bar{q}^{c} q^{c}
$$

The quark masses $M_{q}$ are independent of global c.m. frame time $t$ and of configuration space variables $\vec{z}_{v} ; v=1,2, \cdots, N-1$; the mass functions $m_{q}$ do depend on the entire spacial configurarion of the (resonance-) state considered - always reduced to the overall c.m. system - and therein of time $t$.

For the dynamical meaning of c.m. time the implication of the last relation in eq. 5 repeated below

$$
\vec{\pi}_{N}=N^{-1 / 2} \sum_{\alpha=1}^{N} \vec{p}_{\alpha} \rightarrow 0 \quad, \quad \vec{z}_{N}=N^{-1 / 2} \sum_{\alpha=1}^{N} \vec{x}_{\alpha} \rightarrow 0
$$

is central : the 'prima facie' canonical pair $\vec{\pi}_{N}, \vec{z}_{N}$ in eq. 7 is removed from the set of dynamical canonical (3-vector-)variable pairs

$$
\left(\vec{\pi}_{1}, \vec{z}_{1}\right), \cdots,\left(\vec{\pi}_{N-1}, \vec{z}_{N-1}\right) ; \vec{\pi}_{N}=0, \vec{z}_{N}=0
$$


maintaining kinematic simultaneous vanishing displayed in eq. 8 .

Configuration space variables $\vec{x}_{v} ; v=1, \cdots N$ inherit from the relations displayed in eqs. 7 and 8 the following c.m.-equivalence holds

$$
\vec{x}_{v} \underset{c . m .}{\sim} \vec{x}_{v}-\vec{X} ; \quad v=1, \cdots, N ; \vec{X}=\frac{1}{N}\left(\sum_{\varrho=1}^{N} \vec{x}_{\varrho}\right)
$$

which implies that each $\vec{x}_{v}$ is equivalent to a homogeneous linear combination of the barycentric coordinates $\vec{z}_{\kappa} ; 1 \leq \kappa \leq N-1$. c.m. time $t$ is defined as common time coordinate of four vectors

$$
\begin{aligned}
& \vec{x}_{v} \rightarrow\left\{x^{\mu}\right\}_{v}=\left(t, \vec{x}_{v}\right) ; \quad \begin{array}{l}
\mu=0,1,2,3 \\
v=1, \cdots, N
\end{array} \\
& \text { consistent with the constraint : } \sum_{v=1}^{N} \vec{x}_{v}=0 ; \\
& \text { remembering } N=N_{c}
\end{aligned}
$$

\subsection{Orbits in configuration space and velocities}

The configuration space variables obeying c.m.-constraints and c.m. equivalence are together with c.m. time, as displayed in eq. 10

$$
\left(t, \vec{x}_{1}\right),\left(t, \vec{x}_{2}\right), \cdots,\left(t, \vec{x}_{N}\right) ; \sum_{\varrho=1}^{N} \vec{x}_{\varrho}=0
$$

with $\vec{x}_{v} ; v=1, \cdots, N$ conceived independent of $\mathrm{t}$ - whereas an orbit is formed by binding the space-variables $\vec{x}_{v}=f_{v}(t) \equiv \vec{x}_{v}(t)$, as expressed in the next equation

$$
\begin{aligned}
& \left(t, \vec{x}_{1}\right) \quad,\left(t, \vec{x}_{2}\right) \quad, \cdots,\left(t, \vec{x}_{N}\right) \quad ; \sum_{\varrho=1}^{N} \vec{x}_{\varrho}=0 \\
& \left(t, \vec{x}_{1}(t)\right),\left(t, \vec{x}_{2}(t)\right), \cdots,\left(t, \vec{x}_{N}(t)\right) ; \sum_{\varrho=1}^{N} \vec{x}_{\varrho}(t)=0
\end{aligned}
$$

The definition of orbit in the second relation in eq. 12 leads to the definition of velocities maintaining c.m.-constraints and c.m.-equivalence

$$
\begin{aligned}
& \vec{v}_{v}(t)=\frac{d}{d t} \vec{x}_{v}(t) \equiv \dot{\vec{x}}_{v} ; v=1, \cdots, N ; \\
& \text { with }: \sum_{\varrho=1}^{N} \vec{v}_{\varrho}(t)=0
\end{aligned}
$$

\subsection{The main quantities $\mathcal{L}_{N}$ depends on}

These quantities are compiled in the list of 3 items below

1) a universal scale parameter of dimension mass-square $\Lambda=\left(2 \alpha^{\prime}\right)^{-1}$ related to the inverse slope of Regge trajectories, as well as quark masses, minimally $M_{q} ; q=u, d, s$, composing the mass term in the local QCD Lagrangean given in eq. 6 , where the finite renormalization constants $z_{q}, z_{m}$ are to be chosen to implement exact Ward identities, which in turn specify $M_{q}-$ refs. [20], [21].

2) quark mass functions $m_{\varrho}\left(M_{q} ;\left\{\vec{z}_{v}\right\}\right) ; v=1, \cdots, N-1 ; \varrho=1, \cdots, N$ 
3) velocities $\vec{v}_{v}(t) \equiv \dot{\vec{x}}_{v} ; v=1, \cdots, N$; with : $\sum_{\varrho=1}^{N} \vec{v}_{\varrho}(t)=0$

This leads to the Ansatz - ref. [8] - for light and heavy quark flavors remembering that we use rational units with $\hbar=c=1$.

$$
\begin{aligned}
& \mathcal{L}_{N}=-\sum_{\alpha=1}^{N}\left[m_{\alpha}^{2}-\sum_{\beta=1}^{N} \sum_{\gamma=1}^{N} Q_{\beta \gamma}^{\alpha} \vec{v}_{\beta} \vec{v}_{\gamma}\right]^{1 / 2} \\
& m_{\alpha}=m_{\alpha}\left(M_{\delta}, \Lambda ; \underline{z}\right) ; Q_{\beta \gamma}^{\alpha}=Q_{\beta \gamma}^{\alpha}\left(M_{\delta}, \Lambda ; \underline{z}\right) \\
& \underline{z}=\left\{\vec{z}_{1}, \cdots, \vec{z}_{N-1}\right\}
\end{aligned}
$$

The limiting quantities in the chiral limit obtain as follows

$$
\begin{aligned}
& m_{\alpha}\left(M_{\delta}, \Lambda ; \underline{z}\right) \sim \stackrel{\circ}{m}_{\alpha}(\Lambda ; \underline{z})+M_{\alpha}+o\left(M_{\delta}\right) \\
& Q_{\beta \gamma}^{\alpha}\left(M_{\delta}, \Lambda ; \underline{z}\right) \sim \stackrel{\circ}{Q}_{\beta \gamma}^{\alpha}(\Lambda ; \underline{z})+M_{\varrho} A_{\beta \gamma}^{\varrho \mid \alpha} \Lambda^{\frac{1}{2}}+o\left(M_{\delta} \Lambda^{\frac{1}{2}}\right) \\
& \text { for } M_{\delta} \rightarrow 0
\end{aligned}
$$

In ref. [8] the quantities

$$
\begin{array}{ccc}
m_{\alpha}\left(M_{\delta}, \Lambda ; \underline{z}\right) & \underset{\stackrel{\circ}{m}_{\alpha}}{\longrightarrow}(\Lambda ; \underline{z}) \\
& \text { chiral limit } & \\
Q_{\beta \gamma}^{\alpha}\left(M_{\delta}, \Lambda ; \underline{z}\right) & \longrightarrow & \stackrel{\circ}{Q}_{\beta \gamma}^{\alpha}(\Lambda ; \underline{z})
\end{array}
$$

defined in eq. 15 were identified but shall be kept distinct here, for clarity of logic.

\subsection{Remarks with respect to the chiral limit}

$\chi 1$ The discussion of oscillatory modes of baryons in the chiral limit is simplified but the phenomenological application is limited to the light flavors $\mathrm{u}, \mathrm{d}, \mathrm{s}$.

$\chi 2$ In the zero width approximation the appearance of 8 massless pseudoscalar goldstone bosons

$$
\begin{aligned}
& \stackrel{\circ}{\pi}_{\sigma}=\left(\pi^{+}, \pi^{0}, \pi^{-} ; \bar{K}^{0}, \bar{K}^{-} ; K^{+}, K^{0} ; \eta\right) ; \\
& \sigma=1, \cdots, 8 ; m_{\AA_{\sigma}}=0
\end{aligned}
$$

does not enter directly, but the modes with large masses will decay to lower ones and one ore more $\stackrel{\circ}{\pi}-\mathrm{s}-$ also in cascades. This is after removing infrared divergencies not significantly different for the realistic case of finite $\pi_{\sigma}$ - masses. As a consequence such cascades of excited baryon resonance decays generate a pronounced feeding problem, aggravated if heavy ion collisions are performed at todays energy frontiers at RHIC and LHC, less so at FAIR .

$\chi 3$ In addition at high energy also hevay flavors $\mathrm{c}, \mathrm{b}, \mathrm{t}$ are or will be copiously produced aggravating the study of light flavored

(anti-)baryons .

On the phenomenological side the $\Delta$ Regge trajectory can be found in ref. $22-$ ref. [22] - . The additional trajectories were erased in our subsequent abalysis. 


\section{5. $\mathcal{L}_{N} \rightarrow \stackrel{\circ}{\mathcal{L}}_{N}$ in the chiral limit}

For logical consistency we can consider the chiral limit of arbitrary $N=N_{c}$ and $N_{f l}$ light flavors of quark, as long as $N>1$ and $N>\frac{2}{11} N_{f l}$, ensuring asymptotic ( ultraviolet) freedom .

The Ansatz for $\stackrel{\circ}{\mathcal{L}}_{N}$ becomes

$$
\stackrel{\circ}{Q}_{\beta \gamma}^{\alpha}=\stackrel{\circ}{m}_{\alpha}^{2} \frac{1}{K_{N}} \delta_{\beta \gamma} ; K_{N}: \text { dimensionless, positive constant }
$$

Eq. 14 reduces to

$$
\frac{\stackrel{\circ}{\mathcal{L}}_{N}=-\left(\sum_{\alpha=1}^{N} \stackrel{\circ}{m}_{\alpha}\right)\left[1-\frac{1}{K_{N}} v^{2}\right]^{\frac{1}{2}} ; v^{2}=\sum_{\beta=1}^{N} \vec{v}_{\beta} \vec{v}_{\beta}}{\longrightarrow \stackrel{\circ}{m}=\sum_{\alpha=1}^{N} \stackrel{\circ}{m}_{\alpha} ; \stackrel{\circ}{m}_{\alpha}=\stackrel{\circ}{m}_{\beta}=\stackrel{\circ}{m} / N \quad \forall \alpha, \beta=1, \cdots, N}
$$

In eq. $19 v=\sqrt{v^{2}}$ defines a Euclidean distance in a space over real numbers $R^{\widehat{d}}$ of dimension $\widehat{d}=d_{\text {space }} \times N$, with $d_{\text {space }} \rightarrow 3$, yielding the associated scalar product

$$
\underline{w}=\left\{\vec{w}_{1}, \cdots, \vec{w}_{N}\right\} \rightarrow\left(\underline{v}_{2} \mid \underline{v}_{1}\right)=\sum_{\beta=1}^{N} \vec{v}_{2 \beta} \vec{v}_{1 \beta}
$$

In $R^{\widehat{d}}$ the transformation to barycentric coordinates defined in eq. 5

$$
\begin{aligned}
& \underline{x}=\left\{\vec{x}_{1}, \cdots, \vec{x}_{N}\right\} \rightarrow \underline{z}=\underline{z}(\underline{x})=\left\{\vec{z}_{1}, \cdots, \vec{z}_{N}\right\} \\
& \vec{z}_{1}=\frac{1}{\sqrt{2}}\left(\vec{x}_{1}-\vec{x}_{2}\right) \\
& \vec{z}_{2}=\frac{1}{\sqrt{6}}\left(\vec{x}_{1}+\vec{x}_{2}-2 \vec{x}_{3}\right) \\
& \ldots \\
& \vec{z}_{v}=(v(v+1))^{-1 / 2}\left(\sum_{\alpha=1}^{v}\left(\vec{x}_{\alpha}-\vec{x}_{v+1}\right)\right) \\
& \cdots \\
& \vec{z}_{N-1}=\cdots ;(v=N-1) \\
& \vec{z}_{N}=N^{-1 / 2} \sum_{\alpha=1}^{N} \vec{x}_{\alpha}=N^{1 / 2} \vec{X}_{c . m .}
\end{aligned}
$$

is an orthogonal $O \widehat{d}$ mapping, i.e. preserves the scalar product defined in eq. $20 .^{1}$

The reduction from the $O \widetilde{d}$ transformation, to be proven orthogonal, to its form in $R^{\widetilde{d}}=R^{N} \otimes$ $R^{d_{\text {space }}}$

$$
\begin{aligned}
& O^{(\widehat{d})}=O^{(N)} \otimes \mathbb{I}_{d_{\text {space }} \times d_{\text {space }}} ; O^{(N)} \longleftrightarrow O_{\mu \nu} \\
& \mu, v=1, \cdots, N \text { with } \vec{z}_{\mu}=O_{\mu v} \vec{w}_{v}
\end{aligned}
$$

is not presented here, additional details can be found in ref. [23] and references cited therein.

The proof of orthogonality of $O^{(\widehat{d})}$ reduces to establish orthogonality of the matrix $O^{(N)}$ displayed in eq. 22 .

1 in the approximately realistic case we have $N \equiv N_{c}=d_{\text {space }}=N_{f l}$ $=3$ exactly. 
Two relations follow independently from the c.m. constraint in configuration space $\vec{X}_{c . m .} \sim 0$ from eqs. $18-22$

$$
\begin{aligned}
\sum_{\alpha=1}^{N} \vec{x}_{\alpha}^{2} & =\quad \sum_{\beta=1}^{N-1} \vec{z}_{\beta}^{2}+N \vec{X}_{c . m .}^{2} \\
& =\sum_{\alpha=1}^{N}\left(\vec{x}_{\alpha}-\vec{X}_{c . m .}\right)^{2}+N \vec{X}_{c . m .}^{2} \\
\longrightarrow \sum_{\beta=1}^{N-1} \vec{z}_{\beta}^{2} & =\sum_{\alpha=1}^{N}\left(\vec{x}_{\alpha}-\vec{X}_{c . m .}\right)^{2}
\end{aligned}
$$

\section{6. $\stackrel{\circ}{\mathcal{L}}_{N} \longrightarrow$ constructing canonical variables of Hamiltonian quantum mechanics}

$$
\begin{aligned}
& \vec{p}_{\beta}=\stackrel{\circ}{\mathcal{L}}_{N, \vec{v}_{\beta}}=\frac{\stackrel{\circ}{m}}{K_{N}}\left[1-\omega^{2}\right]^{-1 / 2} \frac{1}{2} v_{, \vec{v}_{\beta}}^{2} \\
& =\frac{\stackrel{\circ}{m}}{K_{N}}\left[1-\omega^{2}\right]^{-1 / 2} \vec{v}_{\beta} \\
& \beta=1, \cdots . N \\
& \omega^{2}=\frac{1}{K_{N}} \sum_{\gamma=1}^{N} v_{\gamma}^{2} ; K_{N}=\frac{N}{N-1} ; \\
& \stackrel{\circ}{m}=\sum_{\alpha=1}^{N} \stackrel{\circ}{m}_{\alpha}
\end{aligned}
$$

Eq. 24 establishes the $\mathrm{N}$ canonically conjugate pairs of $d_{\text {space }}$-vectors

$$
\left\{\vec{p}_{\beta}, \vec{x}_{\beta}\right\} ; \beta=1, \cdots, N
$$

generated through $\stackrel{\circ}{\mathcal{L}}_{N}$ with general properties outlined in eq. 19. These variables do not satisfy the c.m. equivalence $\longrightarrow$ constraints defined in eqs. 7 - 9 collected in eq. 26 below

$$
\begin{aligned}
& \vec{\pi}_{N}=N^{-1 / 2} \sum_{\alpha=1}^{N} \vec{p}_{\alpha} \rightarrow 0, \vec{z}_{N}=N^{-1 / 2} \sum_{\alpha=1}^{N} \vec{x}_{\alpha} \rightarrow 0 \\
& \left(\vec{\pi}_{1}, \vec{z}_{1}\right), \cdots,\left(\vec{\pi}_{N-1}, \vec{z}_{N-1}\right) ; \vec{\pi}_{N}=0, \vec{z}_{N}=0 \\
& \vec{x}_{v} \underset{\text { c.m. }}{\sim} \vec{x}_{v}-\vec{X} ; v=1, \cdots, N ; \vec{X}=\frac{1}{N}\left(\sum_{\varrho=1}^{N} \vec{x}_{\varrho}\right)
\end{aligned}
$$

Omitting many details, we proceed to construct the main conserved quantity, following the original ref. 8 - ref. [8]

$$
\begin{aligned}
& \mathcal{H}_{N}=\sum_{\alpha=1}^{N} \vec{v}_{\alpha} \vec{p}_{\alpha}-\stackrel{\circ}{\mathcal{L}}_{N} ; \sum_{\alpha=1}^{N} \vec{v}_{\alpha} \vec{p}_{\alpha}=\omega^{2}\left[1-\omega^{2}\right]^{-1 / 2} \\
& \stackrel{\circ}{\mathcal{L}}_{N}=-\stackrel{\circ}{m}\left[1-\omega^{2}\right]^{1 / 2} \longrightarrow \mathcal{H}_{N}=\stackrel{\circ}{m}\left[1-\omega^{2}\right]^{-1 / 2}
\end{aligned}
$$

From eqs. 24 and 27 we infer

$$
K_{N} \vec{p}_{\beta}^{2}=\mathcal{H}_{N}^{2} \frac{1}{K_{N}} \vec{v}_{\beta}^{2} \longrightarrow \begin{aligned}
K_{N} \sum_{\gamma=1}^{N} \vec{p}_{\gamma}^{2} & =\mathcal{H}_{N}^{2} \omega^{2} \\
& =\mathcal{H}_{N}^{2}-\stackrel{\circ}{m}^{2}
\end{aligned}
$$

The last relation on the lower right side of eq. 28 follows from the decomposition $\omega^{2}=1-$ $\left(1-\omega^{2}\right)$ and eqs. 24 and 27 . The structure of a genuine harmonic oscillator is brought a step nearer rearranging eq. 28

$$
K_{N} \sum_{\gamma=1}^{N} \vec{p}_{\gamma}^{2}+\stackrel{\circ}{m}^{2}=\mathcal{H}_{N}^{2}
$$


Since eq. 29 derives from a variational principle and yields consistent equations of motion both in the classical as well as the quantized interpretation the Ansatz for $\stackrel{\circ}{m}^{2}$ realizing a system of $s=(N-1) d_{\text {space }}$ linear harmonic oscillators ${ }^{2}$

implies the choice

$$
\begin{aligned}
& \stackrel{\circ}{m}^{2} \propto \sum_{\gamma=1}^{N}\left(\vec{x}_{\beta}-\vec{X}_{c . m .}\right)^{2}+\delta_{m^{2}} ; \\
& \delta_{m^{2}}: \text { configuration space independent }
\end{aligned}
$$

The constant transforming the proportionality in eq. 30 into an equality has to be determined in agreement with canonically conjugate momenta and positions .

The result is, using also the relations in eq. 23

$$
\begin{aligned}
\mathcal{H}_{N}^{2} & =\left[\begin{array}{ll}
\left.\left.K_{N} \sum_{\alpha=1}^{N}\left(\vec{p}_{\alpha}\right)^{2}\right|_{\sum_{\beta=1}^{N} \vec{p}_{\beta}=0}+\stackrel{\circ}{m}^{2}\left(x_{\gamma}-X\right)\right] \\
= \\
+\left.\frac{\left.\Lambda_{N} \sum_{\alpha=1}^{N}\left(\vec{p}_{\alpha}\right)^{2}\right|_{\sum_{\beta=1}^{N} \vec{p}_{\beta}=0}+}{K_{N}} \sum_{\alpha=1}^{N}\left(\vec{x}_{\alpha}\right)^{2}\right|_{\sum_{\beta=1}^{N} \vec{x}_{\beta}=0}+\delta_{m^{2}}
\end{array}\right]
\end{aligned}
$$

Eq. 31 reveals that $\mathcal{H}_{N}^{2}$ so defined does satisfy the c.m.-constraints, and thus by Poincaré invariance is the mass-square operator, henceforth denoted $\mathcal{M}^{2} \equiv \stackrel{\circ}{\mathcal{M}}^{2}$. For simplicity of notation the superfix $\circ$, which stands for the chiral limit, in $\mathcal{M}^{2}$, is suppressed .

Next we transform momenta and positions to barycentric coordinates and cast the relations in eqs. 29 - 31 into the form

$$
\stackrel{\circ}{m}^{2}\left(x_{\gamma}-X\right)=\frac{\Lambda^{2}}{K_{N}} \sum_{\alpha=1}^{N-1} \vec{z}_{\alpha}^{2}+\delta_{m^{2}}
$$

In eq. $32 \Lambda$, of dimension mass-square, is the spring-tension ${ }^{3}$.

$\Lambda^{2}$ is related to the gauge boson condensate of QCD; a detailed discussion can be found in refs. $[19,23]$.

We arrive at the relation for $\mathcal{M}^{2}$ expressed through

$\widehat{d}_{c . m}=(N-1) d_{\text {space }}$ unconstrained, canonically conjugate pairs

$\left(\vec{\pi}_{1}, \vec{z}_{1}\right), \cdots,\left(\vec{\pi}_{N-1}, \vec{z}_{N-1}\right)$ defined in eq. 5

$$
\begin{aligned}
& \mathcal{M}^{2}=\left[K_{N} \sum_{\alpha=1}^{N-1}\left(\vec{\pi}_{\alpha}\right)^{2}+\frac{\Lambda^{2}}{K_{N}} \sum_{\alpha=1}^{N-1}\left(\vec{z}_{\alpha}\right)^{2}+\delta_{m^{2}}\right] \\
& \vec{\pi}_{\alpha}=\frac{1}{i} \partial_{\vec{z}_{\alpha}} ; \alpha=1, \cdots, N-1 ;\left[\vec{\pi}_{\beta k}, \vec{z}_{\gamma l}\right]=\frac{1}{i} \delta_{\beta \gamma} \delta_{k m} \mathbb{I} \\
& k, l=1, \cdots, d_{\text {space }}
\end{aligned}
$$

The structure of the canonical variables in eq. 33 is intrinsically related to the trace anomaly in QCD, as derived in ref. [23], [24].

\footnotetext{
2 For the realistic case $N=d_{\text {space }}=3, \mathrm{~s}$ is $=6$.

3 Spring is not string.
} 


\section{Conclusions and outlook}

In the present paper we calculated the density of states for baryons and compared it to our previous calculation for mesons [17]. The counting of baryons is done here for the first time in a complete fashion and for the mesons it has been published in a previous paper [17]. This calculation is valid in the limit of large $\mathrm{N}$. It includes only oscillatory modes for $\mathrm{u}, \mathrm{d}$,s quarks and antiquarks for $q q q$ baryons and $q \bar{q}$, i.e. is not including exotics and heavy quarks. Furthermore the here derived formulae do not include the intercept and assume a universal slope of the Regge trajectory. Within this approximation all Regge trajectory intercepts are neglected. This however does not affect the counting of the number of these states. For $\mathrm{N}=0$ we find 36 states of mesons and 56 states of baryons as expected from more general Quark Model considerations for u,d,s quarks and antiquarks, and the 56 states of the baryon octet with $\operatorname{spin}=1 / 2$ and the baryon decuplet with $\operatorname{spin}=3 / 2$.

The density of states of baryons and mesons rise as a power law following the construction of oscillatory modes of quarks. We observe that the baryon density of states grows faster than the meson density and is always larger than the latter. The mass square density for $q q q$ baryons and $q \bar{q}$ mesons within the oscillatory modes approach goes like a power determined by the number of independent oscillators and the leading powers are 5 for baryons and 2 for mesons.

The calculation predicts the density of baryonic states including the missing states and does not grow exponentially as in Hagedorn's model, with respect to $\mathrm{N}$ (or mass-square). The fact that there is no limiting temperature in the oscillatory modes approach does not mean that there is no critical temperature for the QCD phase transition .

The estimate of missing hadron states is important to take into account in thermal models that fit the hadron yields and ratios in heavy ion collisions to extract the temperature and chemical potentials of the particle source. Such fits have been performed by many groups for example in references [25], [26], [27], [28], [29].

Furthermore, not only there are missing states of qqbar mesons and $q q q$ baryons and their antiparticles, but probably also missing states of glueballs, tetraquarks, pentaquarks etc., which also influence the feeding and the thermal fits, while there are candidates for non excited glueball states, and strong candidates for tetraquarks and pentaquarks.

An aim for the future would be therefore to obtain main consequences from including the missing hadronic states for the description of thermal equilibrium as applying to the hadronic phase of QCD . A development of the current model would be to proceed along the path of counting oscillatory modes of heavy flavored $q q q$ and $q \bar{q}$ hadrons as well as for multiplets for exotic states like tetraquarks, pentaquarks and glueballsi .

\section{Acknowledgments}

We would like to warmly thank the members of the Theory Division of CERN, and especially Dr. Gian Giudice, Dr Wolfgang Lerche, Prof. Ignatios Antoniades and Dr Luis Alvarez-Gaume, as Theory group leaders, and Ms. Elena Gianolio, Ms. Anne-Marie Perrin, Ms. Michelle Connor and Ms. Jeanne Rostant for their support. We also thank Prof. Moshe Moshe from the Technion University in Haifa, Israel for the information on the paper [30] .

\section{References}

[1] P. Minkowski and F. Halzen, 'Regge Parametrization for $\pi \mathcal{N}$ Scattering', Il Nuovo Cimento, Vol. 1A, N. 1, 1. January 1971, received 1.July 1970 .

[2] R.P. Feynman, M. Kislinger, F. Ravndal, Phys. Rev. D, vol. 3, number 11, 1 june 1971, p. 2706.

[3] K. G. Wilson, 'Confinement of quarks', Phys. Rev. D 10, 2445, 15. Oct. 1974 . 
[4] J. Schwinger, 'A Ninth Baryon ?', Phys. Rev. Lett. 12 (1964) 237 .

[5] Roger F. Dashen, Murray Gell-Mann (Caltech), 1965, 'Approximate symmetry and the algebra of current components', Phys.Lett. 17 (1965) 142-145, DOI: 10.1016/0031-9163(65)90277-5 .

[6] G. Zweig, "Meson classification in the quark model", in "Meson Spectroscopy", edited by C. Baltay and A. H. Rosenfeld, 1968 , 485-496 .

[7] P. Minkowski, Asymptotic freedom-infrared instability, Bern University preprint BE-78-0360, September 1978, 24. pp., in New phenomena in lepton-hadron physics, ed. D. Fries and J. Wess (Plenum Press, New York, 1979) p. 315.

[8] P. Minkowski, 'On The Oscillatory Modes Of Quarks In Baryons', Nucl.Phys. B174 (1980) 258-268, DOI: 10.1016/0550-3213(80)90202-3.

[9] J. Beringer et al. (Particle Data Group), Phys. Rev. D 86 (2012) 010001 .

[10] C. Amsler, T. De Grand and B. Krusche, Quark Model, pdg.lbl.gov/2012/reviews/rpp2012-revquark-model.pdf .

[11] R. Hagedorn, Statistical thermodynamics of strong interactions at high energy, Nuovo Cimento Suppl. 3 (1965) 147-186 .

[12] G. Veneziano, 'Construction of a crossing - symmetric, Regge behaved amplitude for linearly rising trajectories', Nuovo Cim. A57 (1968) 190-197.

[13] J. Schwarz , 'Superstring Theory', Physics Reports 89 (1982) 223.

[14] Enrique Alvarez (CERN), M.A.R. Osorio (Madrid, Autonoma U.), 'Superstrings at Finite Temperature', Oct 1986, 22 pp., CERN-TH-4571 , // Phys.Rev. D36 (1987) 1175 .

[15] Steven S. Gubser (Princeton U.), Sergei Gukov (Princeton U. \& Caltech \& CIT-USC), Igor R. Klebanov (Princeton U.), Mukund Rangamani (Princeton U. \& Caltech \& CIT-USC), Edward Witten (Caltech \& CIT-USC \& Princeton, Inst. Advanced Study), 'The Hagedorn transition in non-commutative open string theory', Sep 2000, PUPT-1949, CALT-68-2296, CITUSC-00-050, J.Math.Phys. 42 (2001) 2749-2764.

[16] U. Heinz et al., 'The Hot QCD White Paper: Exploring the Phases of QCD at RHIC and the LHC', A White Paper on Future Opportunities in Relativistic Heavy Ion Physics, Yasuyuki Akiba (Nishina Ctr., RIKEN), Aaron Angerami (Columbia U.), Helen Caines (Yale U.), Anthony Frawley (Florida State U.), Ulrich Heinz (Ohio State U.), Barbara Jacak (UC, Berkeley), Jiangyong Jia (SUNY, Stony Brook, Chem. Dept.), Tuomas Lappi (Jyvaskyla U.), Wei Li (Rice U.), Abhijit Majumder (Wayne State U.), David Morrison (Brookhaven), Mateusz Ploskon (LBNL, NSD), Joern Putschke (Wayne State U.), Krishna Rajagopal (MIT), Ralf Rapp (Texas A-M), Gunther Roland (MIT), Paul Sorensen (Brookhaven), Urs Wiedemann (CERN), Nu Xu (LBL, Berkeley), W.A. Zajc (Columbia U.), arXiv:1502.02730 [nucl-ex] .

[17] 'Counting of oscillatory modes of valence quarks forming q-qbar mesons', Sonia Kabana, Peter Minkowski, Int.J.Mod.Phys. A31 (2016) no.07, 1650023.

[18] H. Weyl, 'The Theory of Groups and Quantum Mechanics', Dover Books on Mathematics , June 1, 1950 amazon.com

[19] P. Minkowski and S. Kabana, Oscillatory modes of quarks in baryons for 3 quark flavors u,d,s, EPJ Web of Conferences, Volume 71, 2014, 2nd International Conference on New Frontiers in Physics, ICNFP2013.

[20] P. Minkowski, 'Light quark mass ratios (mu:md:ms) from meson and bayyon mass splittings', Modern Physics Letters A Vol. 28, No. 25 (2013) 1360015 and references cited therein.

[21] J. H. Kühn, 'Precise heavy quark masses', Modern Physics Letters A 28, No. 25 (2013) 1360019.

[22] Shuchi Bisht, Navjot Hothi $\dagger$ and Gaurav Bhakuni, 'Phenomenological Analysis of Hadronic Regge Trajectories', EJTP 7, No. 24 (2010) 299-318. 
[23] P. Minkowski, Embedding oscillatory modes of quarks in baryons in QCD, ccsem.infn.it/issp2013/index.html,and references cited therein.

[24] P. Minkowski, On the anomalous divergence of the dilatation current in gauge theories, Bern preprint 1976, URL: mink.itp.unibe.ch .

[25] Mapping out the QCD phase transition in multiparticle production, Sonia Kabana, Peter Minkowski (Bern U.), Oct 2000, 53 pp., New J.Phys. 3 (2001) 4, BUHE-00-09, BUTP-2000-26, DOI: 10.1088/1367-2630/3/1/ 304, e-Print: hep-ph/0010247 .

[26] The Strange border of the QCD phases, Sonia Kabana (Bern U.). Mar 2001. 36 pp. Published in Eur.Phys.J. C21 (2001) 545-555 BUHE-01-01, DOI: 10.1007/s100520100728, e-Print: hep$\mathrm{ph} / 0104001$.

[27] J. Stachel (Heidelberg U.), A. Andronic (Darmstadt, EMMI), P. Braun-Munzinger (Darmstadt, EMMI \& Darmstadt, Tech. U. \& Frankfurt U., FIAS), K. Redlich (Wroclaw U.), 'Confronting LHC data with the statistical hadronization model', Nov 19, 2013. 2 pp., J.Phys.Conf.Ser. 509 (2014) 012019, DOI: 10.1088/1742-6596/509/1/012019, Conference: C13-07-22.1 Proceedings, arXiv:1311.4662 .

[28] Andronic A, Braun-Munzinger P and Stachel J, Dec 2008, 9 pp., Thermal hadron production in relativistic nuclear collisions: The Hadron mass spectrum, the horn, and the QCD phase transition, Phys. Lett. B 673 (2009) 142.

[29] Status of the Thermal Model and Chemical Freeze-Out, J. Cleymans (UCT-CERN Res. Ctr. and Cape Town U.), Dec 22 2014, 5 pp. Published in EPJ Web Conf. 95 (2015) 03004, DOI: 10.1051/epjconf/20159503004, arXiv:1412.7045 [hep-ph].

[30] A Petermann 1965 Nuclear Physics 63349. 